\section{CS-16 REMISSION AND LOW DISEASE ACTIVITY STATE (LDAS) ARE PROTECTIVE OF INTERMEDIATE AND LONG-TERM OUTCOMES IN SLE PATIENTS. DATA FROM A MULTI- ETHNIC, MULTI-CENTER US COHORT}

${ }^{1}$ Graciela S Alarcón*, ${ }^{2,3}$ Manuel Ugarte-Gil, ${ }^{4}$ Guillermo Pons-Estel, ${ }^{5}$ Luis M Vilá, ${ }^{6} J o h n$ D Reveille, ${ }^{1}$ Gerald McGwin Jr. ${ }^{1}$ The University of Alabama at Birmingham, Birmingham, AL, USA; ${ }^{2}$ Hospital Guillermo Almenara, EsSalud, Lima, Perú; ${ }^{3}$ Universidad Cientifica del Sur, Lima, Perú; ${ }^{4}$ Hospital Provincial de Rosario, Argentina; ${ }^{5}$ University of Puerto Rico Medical Sciences Campus; ${ }^{6}$ The University of Texas Health McGovern Medical School

\subsection{6/lupus-2018-Ism.51}

Background Over the last few years the importance of treating patients with SLE towards achieving either Remission or LDAS (Treat-to-Target approach) has become evident. We have now aimed at determining the beneficial effects of achieving these states in lupus patients from a multi-ethnic, multicenter lupus cohort (LUMINA for Lupus in Minorities: Nature vs Nurture).

Methods The LUMINA cohort was started in 1993 and up to 2009 recruited nearly 600 patients of either Caucasian, African or Hispanic ancestry, at three institutions: Alabama, Texas and Puerto Rico. Visits were performed every 6 months for the first year and yearly thereafter. Socioeconomic, demographic and clinical data were obtained at all visits. Disease activity was ascertained with the Systemic Lupus Activity Measure (SLAM) and disease damage with the SLICC Damage Index (SDI). We have now examined all patients' visits and classified them as corresponding to Remission (SLAM score $=0$ and Prednisone $\leq 5 \mathrm{mg} /$ day and no immunosuppressants), LDAS (SLAM score $\leq 3$, prednisone $<7.5 \mathrm{mg} /$ day, no immunosuppressants) or neither: active. Because of the relatively small number of visits corresponding to Remission, Remission and LDAS visits were examined as a single variable. The association between the last SDI and the percent of time on Remission/ LDAS was modeled using Poisson regression with adjustment for variables known to affect this outcome (age, gender, ethnic/racial group, baseline disease activity and disease damage). In a separate multivariable regression model, mortality, adjusting for variables known to affect this outcome, was the endpoint.

Results Visits for 558 patients (total number of visits: 3979; median number of visits per patient: 6.8, interquartile range 4-6) were examined. The longer patients were on Remission/ LDAS, the less likely they were to accrue damage [Parameter estimate -1.049 (Wald 95\% CI -1.3875 to -0.7043 ), $\mathrm{p}<0.001]$. In terms of mortality the direction of the association was as expected (protective) but statistical significance was not reached [Parameter estimate - 1.1932 (Wald 95\% CI 0.8003 to 2.2228$), p=0.1360]$.

Conclusions The longer lupus patients are in remission/LDAS, the less likely they are to accrue damage. Other significant variables in this analysis, were, as expected, associated with damage (older age, male gender, not being from Puerto Rico, higher disease activity at baseline and higher damage at the baseline visit). Although the direction of the association in terms of mortality was as expected, statistical significance was not reached. These data have implications for the management of patients with lupus regardless of their ethnic/racial background.

Acknowledgements To all LUMINA patients for generously giving their time, and to coordinators and research assistants who made this study possible.

\title{
CS-17 CLINICAL AND IMMUNOLOGICAL PROFILE OF PATIENTS WITH SYSTEMIC LUPUS ERYTHEMATOSUS, ATTENDING THE RHEUMATOLOGY CLINICS AT THE SOUTH-WEST REGIONAL HEALTH AUTHORITY, TRINIDAD
}

1,2 Liane Conyette, ${ }^{1,2}$ Haramnauth Dyaanand*, 'Stefan Beharry. 'Rheumatology Unit, Department of Adult Medicine, San Fernando Teaching Hospital; ${ }^{2}$ Associate Clinical Instructor II, Department of Clinical Medical Sciences, Faculty of Medical Sciences, UWI, St Augustine

\subsection{6/lupus-2018-Ism.52}

Background Many patients suffering from Systemic Lupus Erythematosus (SLE) attend one of four rheumatology clinics at the South-West Regional Health Authority of Trinidad. This study aimed at establishing a profile of these patients. A preliminary analysis of the demographic data of these patients, was presented at the lupus 2016 meeting. This abstract highlights the clinical and immunologic profile of the study population.

Methods This study was a cross-sectional design. During the study period in 2016, patients who were being treated for SLE in the public rheumatology clinics of the South-West Regional Health Authority, who fulfilled the Systemic Lupus International Collaborating Clinics Classification Criteria (SLICC) 2012, were identified. Mixed methods (interviewadministered and medical records data extraction) were used.

Abstract CS-17 Table 1 Clinical and immunologic criteria of SLE patients attending rheumatology clinics in south west regional health authority, 2016

Demographic characteristics $\mathrm{N}(\%)$ or mean \pm standard deviation $(n=141)$

Age

Gender

Female

Clinical Criteria

Acute cutaneous lupus

Chronic cutaneous lupus

Oral or nasopharyngeal ulcerations

Nonscarring alopecia

Synovitis involving two or more joints

Serositis

Renal disorder

*Positive Anti-dsDNA

Neurologic disorder

Hemolytic anemia

Leukopenia or lymphopenia

Thrombocytopenia

Immunologic Criteria

**ANA above laboratory reference range

***Anti-dsDNA antibody level above laboratory

reference range

Anti-Sm presence

Antiphospholipid antibody positive

Low complement

Direct Coombs' test in the absence of hemolytic anemia

*Sample size $n=28$ (Results were not obtained for 4 patients)

**Sample size $\mathrm{n}=140$

*** Sample size $n=139$

$39.8 \pm 13.8$

$136(96.5 \%)$

N (\%)

$57(40.4 \%)$

$9(6.4 \%)$

$30(21.3 \%)$

$55(39.0 \%)$

$120(85.1 \%)$

$18(12.8 \%)$

$32(22.7 \%)$

$22(78.6 \%)$

$18(12.8 \%)$

$6(4.3 \%)$

$30(21.3 \%)$

19 (13.5\%)

N (\%)

139 (99.3\%)

$80(57.6 \%)$

$41(29.1 \%)$

$16(11.3 \%)$

$66(46.8 \%)$

$3(2.1 \%)$ 
Descriptive statistics (frequencies, means and standard deviations) were calculated using SPSS version 12.

Results A total of 141 patients (table 1), who fulfilled the SLICC 2012 Classification Criteria for SLE were enrolled in the study. The mean age of the population was 39.8 with a standard deviation of 13.8. 136 patients (96.5\%) were female. Analysis of the immunologic abnormalities revealed a positive ANA test in 139 (99.3\%), and a positive anti-dsDNA test in $80(57.6 \%)$ of the patients, for whom results were available. Synovitis involving two or more joints was reported by 120 $(85.1 \%)$ of the patients, followed by acute cutaneous lupus 57 $(40.4 \%)$ and non-scarring alopecia 55 (39.0\%). Of the 32 patients with renal involvement, 28 patients had a result for an anti-dsDNA. test, of which 22 (78.6\%) were positive.

Conclusion The female to male ratio was comparable to other Asian populations. Our study population had similar clinical and immunologic findings as compared to published literature. A significant proportion of patients who had renal involvement had a positive Anti-dsDNA test. Future longitudinal studies would allow causal inferences to be made.

Acknowledgement The Authors will like to thank all doctors and clerical staff who worked in the Rheumatology Unit during the study period, for their assistance with data collection. Special thanks to Mr Darien Wong and Mr Jared Ramkissoon who assisted with the database. Thanks to Dr Peter Poon King and all consultants at the SWRHA.

\section{CS-18 CEREBROVASCULAR EVENTS IN SYSTEMIC LUPUS ERYTHEMATOSUS: RESULTS FROM AN INTERNATIONAL, INCEPTION COHORT STUDY}

John G Hanly*. Dalhousie University and Queen Elizabeth II Health Sciences Center, Halifax, Nova Scotia, Canada

\subsection{6/lupus-2018-Ism.53}

Background Vascular disease, including involvement of the cerebral circulation, is a frequent cause of morbidity and mortality in SLE. Cerebrovascular events (CerVEs) are reported in $5-18 \%$ of patients in previous cohort studies. Potential etiologies include procoagulant factors due to SLE (e.g. antiphospholipid antibodies, endothelial activation and vasculitis) and factors which promote accelerated atherosclerosis (e.g. hypertension, hyperlipidemia and SLE itself). The relative contribution of these factors and the outcome of clinical CerVEs in a general lupus population have not been well documented.

Objective To determine the frequency, associations and outcomes of cerebrovascular events (CerVEs) in a multi-ethnic/ racial, prospective, SLE disease inception cohort.

Methods Patients were assessed annually for 19 neuropsychiatric (NP) events including 5 types of CerVEs: (i) Stroke; (ii) Transient ischemia; (iii) Chronic multifocal ischemia; (iv) Subarachnoid/intracranial hemorrhage; (v) Sinus thrombosis. Global disease activity (SLEDAI-2K), SLICC/ACR damage index (SDI) and SF-36 scores were collected. Time to event, linear and logistic regressions and multi-state models were used as appropriate.

Results Of 1,826 SLE patients, $88.8 \%$ were female, $48.8 \%$ Caucasian, mean \pm SD age $35.1 \pm 13.3$ years, disease duration $5.6 \pm 4.2$ months and follow-up $6.6 \pm 4.1$ years. CerVEs were the fourth most frequent NP event: 82/1,826 (4.5\%) patients had 109 events, 103/109 (94.5\%) were attributed to SLE and 44/109 (40.4\%) were identified at enrollment. The predominant events were stroke [60/109 (55.0\%)] and transient ischemia [28/109 (25.7\%)]. CerVEs were associated with other NP events attributed to SLE (HR (95\% CI): (3.16; $1.73-5.75) \quad(\mathrm{p}<0.001)$, non-SLE NP $(2.60 ; 1.49-4.51)$ $(\mathrm{p}<0.001)$, African ancestry at US SLICC sites $(2.04 ; 1.01-$ 4.13) $(p=0.047)$ and organ damage $(p=0.041)$. Lupus anticoagulant increased the risk of first stroke and sinus thrombosis $[2.23(1.11,4.45) \mathrm{p}=0.024]$ and TIA $[3.01 \quad(1.15,7.90)$ $\mathrm{p}=0.025]$. Physician assessment indicated resolution or improvement in the majority but patients reported sustained reduction in SF-36 summary and subscale scores following CerVEs $(\mathrm{P}<0.0001)$.

Conclusion CerVEs, the fourth most frequent NP event in SLE, are usually attributable to lupus. In contrast to good physician reported outcomes, patients report a sustained reduction in health-related quality of life following CerVEs.

Acknowledgements This work is presented on behalf of the Systemic Lupus International Collaborating Clinics (SLICC) and was funded in part by a grant from the Canadian Institutes of Health Research (MOP-88526) to Dr. Hanly.

\section{CS-19 HEART FAILURE HOSPITALIZATIONS AMONG SLE AND DIABETES MELLITUS PATIENTS COMPARED TO THE GENERAL U.S. MEDICAID POPULATION}

${ }^{1}$ Sarah K Chen, ${ }^{1}$ Medha Barbhaiya, ${ }^{3}$ Michael A Fischer, ${ }^{1}$ Hongshu Guan, ${ }^{1}$ Candace H Feldman, ${ }^{2}$ Brendan M Everett, ${ }^{1}$ Karen H Costenbader*. 'Divisions of Rheumatology, Immunology and Allergy, Department of Medicine, Brigham and Women's Hospital, Harvard Medical School, Boston, MA; ${ }^{2}$ Pharmacoepidemiology, Department of Medicine, Brigham and Women's Hospital, Harvard Medical School, Boston, MA; ${ }^{3}$ Cardiology, Department of Medicine, Brigham and Women's Hospital, Harvard Medical School, Boston, MA

\subsection{6/lupus-2018-Ism.54}

Background Both SLE and diabetes mellitus (DM) patients have elevated risks of atherosclerotic cardiovascular disease. Risk of heart failure (HF), an end-stage of cardiovascular disease and a leading cause of hospitalization in the U.S., is also elevated among DM patients, but has not been well studied in SLE. We investigated rates and risks of HF hospitalization among SLE patients compared to age- and sex-matched DM and general Medicaid patients.

Methods We used Medicaid Analytic eXtract (MAX) data, containing billing claims for Medicaid patients from the 29 most populated US states 2007-2010. We identified SLE and DM patients, ages 18-65, using $\geq 3$ ICD-9 codes for SLE or DM, each separated by $\geq 30$ days. Index date was $3 \mathrm{rd}$ diagnosis code. We matched each SLE patient at index date to $2 \mathrm{DM}$ patients and 4 general Medicaid patients without SLE or DM, by age at index date and sex. Baseline period was 6 months of continuous Medicaid enrollment prior to index date for all patients. Subjects were followed until death, disenrollment or end of follow-up. We used ICD-9 codes to identify HF hospital discharge diagnosis and calculated rates of first HF hospitalization event per 1000 person-years for each cohort. Cox proportional hazard models, accounting for competing risk of death, estimated hazard ratios (HR) for first HF hospitalization events. In a secondary analysis, we excluded those with baseline HF.

Results 40,212 SLE patients were matched to 80,424 DM and 160848 general patients. In all cohorts, 92\% were female, and mean age was $40.3( \pm 12.1)$ years. Mean follow-up was $1.8( \pm 1.1)$ years for SLE, $1.8( \pm 1.1)$ years for DM, and 1.6 $( \pm 1.2)$ years for general patients. Baseline CVD was present 